\title{
APLICACIÓN DE UN PROGRAMA DE INTERVENCIÓN PARA MEJORAR LA COMPRENSIÓN TÁCTICA EN FÚTBOL SALA: UN ESTUDIO EN CONTEXTO EDUCATIVO
}

\author{
APPLICATION OF AN INTERVENTION PROGRAM TO IMPROVE TACTICAL \\ UNDERSTANDING IN INDOOR FOOTBALL: A STUDY CONDUCTED IN AN \\ EDUCATIONAL CONTEXT
}

\section{A APLICAÇÃO DE UM PROGRAMA DE INTERVENÇÃO PARA MELHORAR A COMPREENSÃO TÁTICA EM FUTSAL: UM ESTUDO REALIZADO NO CONTEXTO EDUCACIONAL}

\section{Alba Práxedes Pizarro* , Luis García-González ${ }^{\star \star *}$, Álvaro Moreno Cortés* M. Perla Moreno Arroyo*, Alberto Moreno Domínguez*}

Keywords

Learning.

Indoor football.

Evaluation.

Resumen: El objetivo del presente estudio fue analizar el efecto de un programa de Enseñanza Comprensiva sobre la toma de decisiones y ejecución del pase y de la conducción en el fútbol sala en un contexto educativo. Participaron 21 alumnos con edades comprendidas entre los 12 y los 14 años. Las variables independientes fueron el programa de intervención basado en el modelo Teaching Games for Understanding (TGFU) y la experiencia en fútbol sala federado. Las variables de toma de decisiones y ejecución fueron evaluadas a través del instrumento de observación Game Performance Evaluation Tool (GPET). Los resultados mostraron una mejoría significativa en la toma de decisión del pase en el grupo sin experiencia tras la aplicación del programa. Sin embargo, en el grupo con experiencia no fueron encontradas diferencias en ninguna de las variables. Los resultados destacan la necesidad de establecer adaptaciones e individualizar las tareas de aprendizaje en el contexto educativo, en función del nivel de habilidad de los alumnos.

\begin{abstract}
The purpose of the study was to analyse the effects of a Comprehensive Teaching Programme on decision-making and execution, pass and dribbling actions in indoor football within an educational context. Participants were 21 students aged 12-14. Independent variables were the intervention program based on the "Teaching Games for Understanding (TGfU)" model and the experience in federated indoor football. Decisionmaking and execution variables were assessed through the observation instrument "Game Performance Evaluation Tool (GPET)". The results show significant improvement in decision making for pass after the application of the program to the unexperienced group; however, those differences were not found in the experienced group. The results point out the need to adjust and individualize learning tasks in the educational context, depending on students' skill level.
\end{abstract}

Palavras-chave Aprendizagem. Futsal. Avaliação.
Resumo: 0 objetivo deste estudo foi analisar o efeito de um Programa de Ensino Compreensivo na tomada de decisões e execução do passe e das ações de drible em futsal num contexto educativo. Participaram 21 alunos com idades entre 12 e 14 anos. As variáveis independentes foram o programa de intervenção baseado no modelo Teaching Games for Understanding (TGFU) e a experiência no futsal federado. As variáveis de tomada de decisão e de execução, foram avaliadas através do instrumento de observação Game Performance Evaluation Tool (GPET). Os resultados mostraram uma melhoria significativa na tomada de decisão do passe no grupo sem experiência após a aplicação do programa. No entanto, no grupo com experiência não foram encontradas diferenças em nenhuma das variáveis. Os resultados destacam a necessidade de estabelecer adaptacões e individualizar as tarefas de aprendizagem no contexto educativo, em função do nível de habilidade dos alunos.
*Universidad de Extremadura. Cáceres, España.

E-mail: apraxede@alumnos.unex.es

** Instituto de Educación Secundaria "Albalat". Navalmoral de la Mata. Cáceres, España.

Email: amorenocortes@ hotmail.com

Recebido em: 23-04-2015 Aprovado em: 07-10-2015 


\section{INTRODUCCIÓN}

Durante muchas décadas, la Educación Física (EF) impartida en los centros escolares se centraba en la reproducción de los gestos técnicos en tareas analíticas, el conocimiento de las reglas del juego y la aplicación de lo aprendido en partidos al final de las clases. Este modelo provocaba que los alumnos obtuvieran un escaso conocimiento de la dinámica intrínseca del juego (BUNKER; THORPE, 1982). Por su parte, Wein (2012), en un contexto no muy alejado como es el de la iniciación deportiva, plantea el sugerente reto de formar jugadores inteligentes que sean capaces, entre otras cosas, de manejar varias alternativas tácticas y seleccionar la mejor en poco tiempo, mantener control visual sobre el espacio de juego y la colocación de los jugadores, realizar sus acciones en el momento justo y adaptarse a las situaciones cambiantes.

En este sentido, en las últimas tres décadas, la enseñanza de los deportes ha progresado hacia una metodología basada en la enseñanza comprensiva, que estimula a los alumnos a resolver problemas mediante la utilización de su experiencia previa a medida que se implican en los deportes y actividades similares al mismo (GUTIÉRREZ; GARCÍA-LÓPEZ, 2012, SINGLETON, 2009). Por ello, los alumnos que tienen una mayor pericia deportiva en este deporte o uno similar (entendiendo similar como un deporte que comparte los mismos principios tácticos de actuación), se caracterizan por tener un conocimiento más sofisticado, que conlleva a desarrollar decisiones tácticamente más adecuadas (NIELSEN; MCPHERSON, 2001).

En contraposición a este modelo, Bunker y Thorpe (1982) critican el modelo tradicional, argumentando que, a través de éste, la mayoría de los alumnos obtienen un escaso conocimiento de la dinámica intrínseca del juego durante las clases de EF, dando como resultado una capacidad limitada para tomar decisiones (para una revisión, ver STOLZ; PILL, 2014) y, una falta de creatividad y reflexión sobre el deporte (HOPPER, 2002). Así, en el modelo de instrucción directa, las condiciones de práctica se descontextualizan, trabajándose la técnica de forma aislada e imposibilitando así, generalizar la práctica a condiciones reales de juego (LIGHT; HARVEY; MOUCHET, 2014). A pesar de ello, este cambio en la enseñanza de los deportes es más evidente en la literatura publicada que en la práctica real (ROBLES; GIMÉNEZ; ABAD, 2011).

Por su parte, Gutiérrez, etal. (2014) señalan que los modelos de enseñanza comprensiva sitúan al alumno en el centro del aprendizaje, haciendo hincapié en el desarrollo de la toma de decisiones, actitud crítica y resolución de problemas. Además este modelo favorece el desarrollo de los aspectos cognitivos, un componente esencial de las clases de EF (CORAL; LLEIXÁ, 2014).

En el paradigma de la psicología cognitiva, Griffin, Brooker y Patton (2005) sitúan al modelo Teaching Games for Understanding (TGfU) desarrollado por Bunker y Thorpe (1982), como una de las metodologías más favorecedoras de la capacidad decisional de los deportistas. Este modelo, ha sido avalado por numerosos autores como un modelo eficaz, tanto en contextos educativos (BALAKRISHNAN; RENGASAMY; AMAN, 2011, HARVEY; GITTINS, 2014, TURNER; MARTINEK, 1992) como en contextos deportivos (HARVEY; CUSHION; MASSA-GONZÁLEZ, 2010), donde los jugadores tienen un nivel de pericia superior (HARVEY, 2003). Sin embargo, no se encuentran estudios que hayan evaluado la efectividad de estos planteamientos en alumnos con diferente nivel de pericia.

El modelo TGfU, basado en los principios pedagógicos del juego modificado (Juego Modificado por Representación "JMR" y Juego Modificado por Exageración "JME") y en el 
cuestionamiento (THORPE; BUNKER; ALMOND, 1986), tiene como objetivo la comprensión del juego a través del conocimiento táctico (GRAY; SPROULE, 2011). Bajo este planteamiento, el juego modificado permite que los contenidos se aprendan siempre en una situación contextual, donde el comportamiento individual del alumno adquiere sentido en una situación táctica de juego real, vivenciando así un número más elevado de situaciones específicas de juego (SERRA; GONZÁLEZ-VÍLLORA; GARCÍA-LÓPEZ, 2011). Por otra parte, el cuestionamiento es una herramienta que ha resultado ser muy útil, ya que provoca mejoras, tanto en la ejecución motriz de una habilidad técnica, como en las variables decisionales y tácticas en deportes de carácter abierto (GIL; DEL VILLAR, 2014).

Sin embargo, el impacto del modelo TGfU en la práctica docente es todavía muy limitado. Analizando la realidad educativa del contexto en el que se ha realizado este estudio, se observa que la enseñanza de la técnica sigue siendo la prioridad en el tiempo de clase y no es frecuente el uso de estilos de enseñanza que promuevan la significatividad y reflexión sobre los aprendizajes (ROBLES et al., 2011) . Este predominio del modelo tradicional también se extiende al ámbito del deporte extraescolar, ya que monitores y técnicos deportivos suelen utilizar una metodología basada en la técnica (ABAD et al., 2011)

Por parte, la evaluación de estas metodologías ha sido objeto de debate, siendo uno de los instrumentos de evaluación más utilizado dentro del ámbito de la EF (HASTIE; SINELNIKOV; GUARINO, 2009) el Game Performance Assessment Instrument (GPAI) (OSLIN; MITCHELL; GRIFFIN, 1998). Estos autores desarrollaron el GPAI para medir los comportamientos del jugador/alumno en el rendimiento del juego, con el fin de demostrar una mayor comprensión táctica, una mejor capacidad del jugador/alumno para resolver problemas tácticos y asícomo, una mejor selección y aplicación de las habilidades apropiadas en cada momento. Específicamente en fútbol, García-López et al. (2013) diseñaron el Game Performance Evaluation Tool (GPET) o Herramienta de Evaluación del Rendimiento del Juego.

Por todo ello, el objetivo de la presente investigación fue evaluar la incidencia de un programa de Enseñanza Comprensiva, basado en el modelo TGfU, sobre la toma de decisiones y la ejecución en las habilidades técnico-tácticas del pase y la conducción en el fútbol sala, en alumnos de secundaria con diferente nivel de pericia.

\section{MÉTODO}

\subsection{Participantes}

La muestra del presente estudio estuvo compuesta por 21 alumnos de dos grupos diferentes de $1^{\circ}$ ESO de un Instituto de Educación Secundaria de la provincia de Cáceres. Para valorar la necesidad de la individualización de la enseñanza en las clases de EF, a la hora de realizar los diferentes análisis estadísticos, los alumnos se agruparon en dos grupos, formando el grupo sin experiencia, 9 alumnos (edad: $12.66 \pm .50$; experiencia: 0 ) y el grupo con experiencia, 12 alumnos (edad: $13 \pm .73$; experiencia: $3.08 \pm 1.24$ ).

Tras la aplicación del programa, no fueron considerados aquellos participantes que faltaron a 2 o más clases, aquellos que no asistieron a la evaluación inicial o a la evaluación final, y aquellos que tenían menos de 5 acciones en algunas de las categorías de codificación del programa de intervención (pase y conducción). 
A nivel ético se siguieron las directrices de la Declaración de Helsinki (2008) con respecto al consentimiento, confidencialidad y anonimato de los resultados.

\subsection{VARIABLES}

\subsubsection{Variables independientes}

La primera variable independiente considerada en la presente investigación se corresponde con el programa de Enseñanza Comprensiva basado en el modelo TGfU para la mejora de la toma de decisiones y ejecución en el fútbol sala.

El programa se llevó a cabo en contexto educativo y estuvo formado por dos sesiones de evaluación (una de evaluación inicial y otra de evaluación final) y 7 de desarrollo o aprendizaje, estando compuesta la unidad didáctica por nueve sesiones. El programa se basó en el modelo Teaching Game for Understanding (TGfU), siendo la característica principal de éste la utilización del cuestionamiento (feedback interrogativo), para que el alumno reflexionara durante la acción y se produjera así una mejora en los mecanismos cognitivos, como es la toma de decisiones.

El programa de intervención fue desarrollado en dos cursos diferentes de 1ํㅡㅇ, siendo el mismo profesor el responsable de ambos grupos, llevándose a cabo en el tercer trimestre, de acuerdo con la temporalidad prevista para este contenido en la programación didáctica del área de EF del centro. Así, los contenidos principales fueron el pase y la conducción, organizadas las sesiones de la siguiente manera: en la primera y segunda se trabajó el pase como contenido principal, en la tercera y cuarta, la conducción, y en la quinta, sexta y séptima, se trabajaron de forma integrada junto con el lanzamiento.

Para el diseño de las actividades del programa de intervención, se manipularon las variables tácticas que permitían modificar el juego e ir progresando en la complejidad de éste (principios del juego, número de alumnos por equipo, nivel de oposición, tamaño del campo y la portería, y la duración del juego). De este modo, inicialmente el número de componentes de un equipo era mínimo, existía superioridad numérica en ataque, el tamaño del terreno de juego era grande, no existía limitación de tiempo para conseguir el objetivo y el tamaño de la portería era mayor. Así, se favorecía la máxima participación, una mayor continuidad en el juego, una menor exigencia táctica y una mayor facilidad para la ejecución técnica.

La segunda variable independiente que se consideró se corresponde con la experiencia en fútbol sala federado. En función de esta variable y únicamente para el análisis estadístico de los datos, los alumnos fueron agrupados, en dos grupos en función de su experiencia en este deporte, identificados como grupo de alumnos sin experiencia en fútbol sala federado y grupo de alumnos con experiencia en fútbol sala federado. El grupo de alumnos sin experiencia no tenían ningún año de experiencia en la práctica del fútbol sala y el grupo de alumnos con experiencia tenían uno o más años de práctica.

\subsubsection{Variables dependientes}

- Toma de decisiones, medida a través del porcentaje de decisiones acertadas. Se registraron el número de acciones apropiadas e inapropiadas del pase y de la conducción, desde el punto de vista decisional, mediante la utilización del GPET (GARCÍA-LÓPEZ et al., 2013), 
considerando para ello la categoría de toma de decisiones. Los valores finales de porcentaje de decisiones adecuadas, para cada alumno en cada una de las situaciones de evaluación, fueron calculados mediante la siguiente fórmula: número de decisiones adecuadas/total de decisiones.

- Ejecución, medida a través del porcentaje de ejecuciones adecuadas. Se registraron el número de acciones apropiadas e inapropiadas del pase y de la conducción, desde el punto de vista de la ejecución, mediante la utilización del GPET (GARCÍA-LÓPEZ et al., 2013), considerando para ello la categoría de ejecución. Los valores finales de porcentaje de ejecuciones adecuadas, para cada alumno en cada una de las situaciones de evaluación, fueron calculados mediante la siguiente fórmula: número de ejecuciones adecuadas/total de ejecuciones.

\subsection{Material e instrumento}

El instrumento de recogida de datos utilizado en el presente estudio fue la observación sistemática de la toma de decisiones y ejecución del pase y la conducción en situaciones de ataque, utilizándose para ello, el GPET (GARCÍA-LÓPEZ et al., 2013). Se registraron todas las acciones de pase y conducción de cada uno de los alumnos que formaban ambos equipos. Para la valoración de la toma de decisiones se utilizó el componente toma de decisiones del citado instrumento, asignando un valor 1 a decisiones apropiadas (e.g. para la acción de pase: pasar a un compañero que está libre de marcaje) y con un 0 a aquellas decisiones inapropiadas (e.g. para la acción de conducción: conducir con el balón cuando un oponente está cerca y tiene oportunidades de ganar el balón). Para la valoración de la ejecución se utilizó el componente ejecución del citado instrumento, asignando un valor 1 a ejecuciones con éxito (e.g. para la acción de conducción: atacar con el balón de forma exitosa) y con un 0 a aquellas ejecuciones sin éxito (e.g. para la acción de pase: el pase es interceptado).

Para garantizar la fiabilidad de la observación, un observador conocedor del fútbol sala, realizó un proceso de entrenamiento, en el que se utilizó una muestra superior al 10\% de la muestra total. En la observación de todas las variables se alcanzaron unos valores de Kappa de Cohen intra-observador superiores a .81, valor a partir del cual se considera una concordancia casi perfecta (FLEISS; LEVI; CHO PAIK, 2003). Para garantizar la fiabilidad temporal de la medida, se desarrolló la misma codificación en dos momentos diferentes, con una diferencia temporal de diez días, obteniendo unos valores de Kappa de Cohen superiores a .85.

Para el desarrollo de esta investigación fueron utilizados los siguientes medios audiovisuales y tecnológicos: un ordenador portátil SONY VAIO, una vídeo-cámara Sony HDRXR155, un trípode Hama Gamma Series y el programa informático para datos estadísticos SPSS 19.0.

\subsection{Procedimiento}

Con el objeto de garantizar la aplicación correcta del modelo de enseñanza comprensiva (TGfU) se aleccionó al docente que iba a desarrollar el programa, por parte de un experto. Al igual que en el estudio de Harvey et al., (2010), el programa de formación se desarrolló durante tres sesiones, de una duración de una hora y media cada una. En la primera de ellas se 
abordaron los principios básicos del modelo TGfU, en la segunda se incidió sobre la aplicación de los juegos modificados y en la última sobre la utilización del cuestionamiento en la formación de los estudiantes. Estas sesiones de formación fueron desarrolladas por el investigador principal con dilatada experiencia y amplio conocimiento del objeto de estudio. Igualmente, durante la primera sesión de desarrollo de la UD, este investigador estuvo presente, en un lugar alejado y sin intervenir en la práctica, con el fin de valorar si los conocimientos adquiridos por el profesor durante la formación fueron adecuados.

Posteriormente, tuvo lugar la evaluación inicial, desarrollándose en dos días diferentes, uno para cada grupo de 1ㅌSO. En cada sesión se jugaban cuatro partidos de $4 \times 4$ (en una pista de fútbol sala de $32 \times 16 \mathrm{~m}$ exterior, pero sin posibilidad de incidencia de otros agentes externos que pudieran interrumpir la práctica) en los que se enfrentaban alumnos con la misma experiencia. De esta forma, todos los alumnos jugaron dos partidos de ocho minutos de duración, con un total de 16 minutos para la evaluación de cada alumno. Para la grabación de los partidos, el profesor utilizó una vídeo-cámara Sony HDR-XR155, situada en una esquina de la pista, a tres metros de altura, garantizando un óptimo plano de visión de todo el terreno de juego. Entre la evaluación inicial y la final, se desarrollaron las siete sesiones de aprendizaje de las que estaba compuesto el programa de intervención. Todos los alumnos desarrollaron las mismas actividades de aprendizaje. Los agrupamientos durante las sesiones fueron organizados por el profesor, en función al desarrollo y evolución de las actividades.

Finalmente, tuvo lugar la evaluación final, que se llevó a cabo siguiendo el mismo procedimiento que la evaluación inicial, enfrentándose así los mismos equipos.

\subsection{Análisis estadístico}

Se utilizó el programa estadístico SPSS 19.0 para el análisis y tratamiento de los datos. Se examinó la normalidad de los datos a través de la prueba de Shapiro-Wilk, como paso previo a la aplicación del programa de intervención. La evaluación de este estadístico determinó el uso de estadística paramétrica.

Por ello, para las variables de toma de decisiones y ejecución, se realizó un análisis inferencial a través de un ANOVA mixto, de dos factores con medidas repetidas en un factor, con el fin de determinar el efecto sobre la interacción entre los dos momentos de medición (preintervención y post-intervención) y entre los dos grupos (sin experiencia y con experiencia). Para determinar cuál era el tamaño del efecto se utilizó el estadístico Eta al cuadrado parcial ( $\eta^{2}$ parcial).

\section{RESULTADOS}

Con respecto a la toma de decisiones, la Tabla 1 muestra las comparaciones por pares en cada uno de los grupos. Como se puede observar, en el grupo sin experiencia, sí existe una diferencia significativa tras el programa de intervención en la acción del pase, no ocurriendo lo mismo en la acción de la conducción. Por lo contrario, en el grupo con experiencia, no se observa diferencia alguna en ninguna de las acciones estudiadas. 
Tabla 1 - Estadísticos descriptivos y comparaciones intra-grupo de la toma de decisiones

\begin{tabular}{|c|c|c|c|c|c|c|c|c|c|c|c|c|}
\hline \multirow[t]{2}{*}{ Medida } & \multirow[t]{2}{*}{ Grupo } & \multicolumn{3}{|c|}{$\begin{array}{c}\text { (l) } \\
\text { Toma de } \\
\text { decisiones }\end{array}$} & \multicolumn{3}{|c|}{$\begin{array}{c}\text { (J) } \\
\text { Toma de decisiones }\end{array}$} & \multirow[t]{2}{*}{$\begin{array}{l}\text { Dif. de } \\
\text { medias } \\
(I-J)\end{array}$} & \multirow[t]{2}{*}{$\begin{array}{l}\text { Error } \\
\text { típ. }\end{array}$} & \multirow[t]{2}{*}{$P$} & \multicolumn{2}{|c|}{$\begin{array}{c}\text { IC 95\% } \\
\text { diferencias }\end{array}$} \\
\hline & & $T^{\prime}$ & $M$ & DT & $\mathrm{T}^{\prime}$ & $M$ & DT & & & & L.I. & L.S. \\
\hline \multirow{2}{*}{ Pase } & Sin & Pre & 43.42 & 29.29 & Post & 66.15 & 16.46 & -22.72 & 10.77 & .050 & -45.46 & .005 \\
\hline & Con & Pre & 73.66 & 16.39 & Post & 66.60 & 16.18 & 9.56 & 8.23 & .261 & -7.79 & 26.93 \\
\hline \multirow{2}{*}{ Conducción } & Sin & Pre & 55.14 & 21.40 & Post & 38.21 & 36.98 & 16.92 & 12.79 & .203 & -10.06 & 43.92 \\
\hline & Con & Pre & 63.64 & 24.65 & Post & 60.74 & 31.72 & 2.90 & 9.77 & .770 & -17.71 & 23.51 \\
\hline
\end{tabular}

Fuente: datos de los autores.

Nota. Sin: grupo sin experiencia. Con: grupo con experiencia. Pre = medida de pre-intervención; Post = medida de post-intervención; L.I. = límite inferior; L.S. = límite superior.

Con respecto a la ejecución, la Tabla 2 muestra las comparaciones por pares en cada uno de los grupos. Como se puede comprobar, en ninguno de los dos grupos se observa una diferencia significativa tras el programa de intervención, en las variables de ejecución del pase y la conducción.

Tabla 2 - Estadísticos descriptivos y comparaciones intra-grupo de la ejecución

\begin{tabular}{|c|c|c|c|c|c|c|c|c|c|c|c|c|}
\hline \multirow[t]{2}{*}{ Medida } & \multirow[t]{2}{*}{ Grupo } & \multicolumn{3}{|c|}{$\begin{array}{c}\text { (l) } \\
\text { Toma de } \\
\text { decisiones }\end{array}$} & \multicolumn{3}{|c|}{$\begin{array}{c}(\mathrm{J}) \\
\text { Toma de decisiones }\end{array}$} & \multirow[t]{2}{*}{$\begin{array}{l}\text { Dif. de } \\
\text { medias } \\
(I-J)\end{array}$} & \multirow[t]{2}{*}{$\begin{array}{l}\text { Error } \\
\text { típ. }\end{array}$} & \multirow[t]{2}{*}{$P$} & \multicolumn{2}{|c|}{$\begin{array}{c}\text { IC 95\% } \\
\text { diferencias }\end{array}$} \\
\hline & & $\mathrm{T}^{\prime}$ & M & DT & $T^{\prime}$ & M & DT & & & & L.I. & L.S. \\
\hline \multirow{2}{*}{ Pase } & $\operatorname{Sin}$ & Pre & 30.67 & 20.33 & Post & 53.30 & 16.52 & -22.62 & 11.53 & .066 & -46.96 & 1.71 \\
\hline & Con & Pre & 66.60 & 16.18 & Post & 60.45 & 24.94 & 6.15 & 8.81 & .495 & -12.44 & 24.96 \\
\hline \multirow{2}{*}{ Conducción } & $\operatorname{Sin}$ & Pre & 47.27 & 21.77 & Post & 41.98 & 29.62 & 5.28 & 14.33 & .717 & -24.95 & 35.52 \\
\hline & Con & Pre & 45.60 & 31.55 & Post & 43.54 & 32.06 & 2.06 & 10.94 & .852 & -21.03 & 25.16 \\
\hline
\end{tabular}

Fuente: datos de los autores.

Nota. Sin: grupo sin experiencia. Con: grupo con experiencia. Pre = medida de pre-intervención; Post = medida de post-intervención; L.I. = límite inferior; L.S. = límite superior.

\section{DISCUSIÓN}

El objetivo de la presente investigación fue evaluar la incidencia de un programa de Enseñanza Comprensiva, basado en el modelo TGfU, sobre la toma de decisiones y la ejecución en las habilidades técnico-tácticas del pase y la conducción en el fútbol sala, en alumnos con diferente nivel de pericia.

En cuanto a la variable de toma de decisiones, los resultados obtenidos muestran diferencias significativas en el grupo sin experiencia en la acción del pase, mejorando así estos alumnos, tras el programa de intervención, la capacidad decisional en esta habilidad. Por el contrario, tales diferencias no se encuentran en la acción de la conducción. Por otro lado, en el grupo con experiencia, no fueron encontradas diferencias tras el programa de intervención en la toma de decisiones en ninguna de las acciones estudiadas.

Estos resultados son congruentes como los obtenidos en otros estudios en los que se aplicó un programa de intervención basado en el modelo TGfU (HARVEY, 2003). 
Los resultados mostraron mejoras en las variables decisionales. Esto se debe a que estos alumnos desarrollaron un conocimiento más sofisticado, que conlleva a desarrollar decisiones tácticamente más adecuadas (NIELSEN; MCPHERSON, 2001).

En este sentido, y de forma general, los alumnos del grupo sin experiencia que fueron sometidos al programa de intervención para la mejora de la toma de decisiones, alcanzaron un nivel de pericia cognitiva superior, desarrollando una selección de la respuesta más táctica en la acción de pase (DEL VILLAR; et al., 2007; GUTIÉRREZ; GONZÁLEZ-VÍLLORA; GARCÍALÓPEZ; MITCHELL, 2011).

En primer lugar, consideramos que al ser la conducción un elemento más técnico que el pase, una metodología comprensiva que se centra en patrones tácticos o cognitivos puede haber incidido en menor medida en este tipo de habilidades. A este respecto, González-Víllora et al. (2011), en su estudio en fútbol, los resultados mostraron que los jugadores tenían más limitaciones en algunos contenidos técnico-tácticos que en otros, como es la finta, elemento fundamental del regate.

Por otra parte, el hecho de que no se encontraran diferencias significativas en el grupo con experiencia, puede ser debido a que las tareas no estuvieran adecuadamente adaptadas al nivel de estos alumnos, valorando así la necesidad de diseñar en función de la experiencia (MAÑERO; RODRÍGUEZ, 2011) y del nivel de habilidad que tengan los alumnos, ya que los aprendizajes serán diferentes en función de ésta (ARAÚJO; MESQUITA; HASTIE, 2014). Es importante señalar que esta diferencia, en cuanto al nivel de habilidad se refiere, puede ser debida a que el fútbol es considerado probablemente como el deporte más popular en el mundo (AGUIAR et al., 2012), provocando así, que aquellos alumnos que lo practican en su tiempo de ocio, lleguen a las clases con un nivel superior que los que no lo practican. A este respecto, Ayvazo y Ward (2011) señalan que un buen docente tiene que ser capaz de modificar las tareas, haciéndolas más fáciles si el alumno no es capaz de conseguir el objetivo o por lo contrario, planteando un reto más difícil si lo lleva a cabo con facilidad.

Por lo tanto, para que el grupo de alumnos con experiencia mejore en las habilidades técnico-tácticas de este deporte, es necesario que las tareas estén adaptadas al nivel de habilidad que posean y a la experiencia que tengan en un deporte con características similares.

En cuanto a la variable de ejecución, los resultados obtenidos no muestran diferencias significativas en ninguno de los dos grupos en ninguna de las acciones estudiadas. Los resultados obtenidos muestran que el programa de Enseñanza Comprensiva aplicado, no ha sido suficientemente eficaz para provocar una mejora en la variable de ejecución, en ninguno de los dos grupos en las acciones del pase y de la conducción.

En la literatura científica, se encuentran estudios similares que demuestran que los alumnos que fueron sometidos al programa de intervención basado en metodologías comprensivas, obtuvieron mejoras significativas en la toma de decisiones, pero no así en la ejecución de la habilidad motriz (TURNER; MARTINEK, 1992). Esto puede ser debido a que, en el contexto educativo, estos programas le dan un mayor énfasis a la mejora de la toma de decisiones, al desarrollo del pensamiento crítico y a la resolución de problemas (GUTIÉRREZ et al., 2014), dejando en un segundo plano factores determinantes del rendimiento como son las variables técnicas (PHILLIPS et al., 2010). En este sentido, el currículo de EF señala que el deporte en el área de EF debe conseguir una AF libre de cualquier aspecto de rendimiento 
y que se debe valorar prioritariamente la toma de decisiones necesaria para la resolución de situaciones de juego reducido más que la ejecución técnica de las habilidades que se desarrollan. Este hecho puede haber propiciado que no hubiera diferencias significativas en la variable de ejecución.

Por otra parte, la duración del programa, escasa, puede ser también otra causante de que no se obtuvieran mejoras en la variable de ejecución. En su estudio, Tallir et al. (2004) exponen que la eficiencia de ésta y la mejora de los alumnos, depende en mayor medida del tiempo, comprobándose en este estudio que duró 12 sesiones, en el que se comparaban metodologías tradicionales y comprensivas en el contexto educativo, ninguna mejora en los alumnos. En este sentido, Bunker y Thorpe (1982) señalan que la técnica, para que pueda desarrollarse de forma eficaz, se debe trabajar más tiempo, con lo que la escasa duración del programa, puede haber favorecido a un desarrollo básico de habilidades tácticas sencillas como la toma de decisiones, pero no al desarrollo de la técnica de estas acciones. De la misma manera, entendemos que, el desarrollo del pensamiento crítico y la conciencia táctica en el alumno debe ser el objetivo fundamental del profesorado en sus programaciones didácticas, y no tanto la mejora de la ejecución.

Por todo ello, los resultados parecen indicar que el uso de metodologías comprensivas en el contexto educativo puede ser eficaz en los alumnos sin experiencia, y en habilidades más abiertas como el pase. No obstante, estos modelos ya se han mostrado eficaz para distintos niveles de pericia deportiva, por lo que consideramos que la individualización debe tenerse en cuenta para que el proceso de enseñanza/aprendizaje sea eficaz para todo el alumnado, indistintamente del nivel de experiencia (CHOW et al., 2009).

Finalmente, consideramos que en futuras investigaciones, ampliar la muestra podría aportar una mayor significatividad a los resultados encontrados. De igual modo, entendemos que la escasa duración del programa puede haber condicionado los resultados, especialmente en la ejecución de la habilidad, por lo que sería interesante ampliar la UD a 9-10 sesiones de desarrollo. Estudios que profundicen en la aplicación de estos programas en alumnos con diferente nivel de pericia podría ayudar a orientar la labor de los docentes de EF.

\section{CONCLUSIONES}

Con respecto a la toma de decisiones, el programa de Enseñanza Comprensiva basado en el modelo TGfU para la mejora de la toma de decisiones y ejecución en el fútbol sala ha influido positivamente en la toma de decisiones del pase en los alumnos sin experiencia, no influyendo de esta misma manera en los alumnos con experiencia. De esta forma, consideramos que el modelo "TGfU" puede ser utilizado como método para la enseñanza de los deportes en la escuela, adaptándose a las características de los alumnos tanto como sea posible, elevando el nivel de exigencia de las tareas para los alumnos que tengan una mayor experiencia en la habilidad a aprender.

Con respecto a la ejecución, el programa no ha incidido en ninguno de los dos grupos de forma positiva ni significativa en esta variable en ninguna de las acciones estudiadas. Podemos considerar entonces, que el aprendizaje de la técnica requiere de un mayor tiempo de enseñanza/aprendizaje, dificultándose la adquisición de ésta con un programa de intervención con reducidas sesiones. Por todo ello, se recomienda orientar la enseñanza de las 
habilidades deportivas en contexto educativo hacia patrones más tácticos y hacia la integración de contenidos por el escaso tiempo de que disponen los profesores de EF para el desarrollo de sus unidades didácticas, aumentando así las posibilidades de incidir en el aprendizaje del alumnado. De igual modo, se podría recomendar la necesidad de incluir en las programaciones didáctica contenidos novedosos, que conozcan poco los alumnos y cuya experiencia sea la misma en todos los alumnos.

\section{REFERENCIAS}

ABAD, Manuel T. et al. Perfil, experiencia y métodos de enseñanza de los entrenadores de jóvenes futbolistas en la provincia de Huelva. Retos: Nuevas Tendencias en Educación Física, Deporte y Recreación, Murcia, v. 20, p. 21-25, 2011.

AGUIAR, Marco et al. A review on the effects of soccer small-sided games. Journal of Human Kinetics, Katowice, v. 33, p. 103-13, jun. 2012.

ARAÚJO, Rui; MESQUITA, Isabel; HASTIE, Peter. A. Review of the Status of Learning in Research on Sport Education: Future Research and Practice. Journal of Sports Science and Medicine, Bursa, v. 13, n. 4, p. 846-858, dec. 2014.

AYVAZO, Shiri; WARD, Phillip. Pedagogical content knowledge of experienced teachers in physical education: functional analysis of adaptations. Research Quarterly for Exercise and Sport, Champaign, v. 82, n. 4, p. 675-684, dec. 2011.

BALAKRISHNAN, Malathi; RENGASAMY, Shabeshan; AMAN, Mohd S. Teaching game for understanding in physical education: A theoretical framework and implication. ATIKAN, Bandung, v. 1, n. 2, p. 201-214, 2011.

BUNKER, David; THORPE, Rod. A model for the teaching of games in secondary schools. Bulletin of Physical Education, London, v. 18, n. 1, p. 5-8, 1982.

CHOW, Jia Y et al. Nonlinear pedagogy: implications for teaching games for understanding (TGfU). In: TGfU: simply good pedagogy, understanding a complex challenge. Vancouver: University of British, 2009. p. 14-17.

CORAL, Josep; LLEIXÁ, Teresa. La enseñanza de la Educación Física mediante el enfoque educativo Clil: la resolución de los dilemas profesionales surgidos durante un proceso de investigación-acción.

Movimento, Porto Alegre, v. 20, n. 4, p. 1447-1472, 2014.

DEL VILLAR, Fernando et al. Expert-novice differences in cognitive and execution skills during tennis competition. Perceptual and Motor Skills, Missoula, v. 104, n. 2, 355-365, 2007.

FLEISS, Joseph L.; LEVI, Bruce; CHO PAIK, Myunghee. Statistical methods for rates and proportions. New York: Wiley, 2003.

GARCÍA-LÓPEZ, Luis M. et al. Development and validation of the Game Performance Evaluation Tool (GPET) in soccer. Revista Euroamericana de Ciencias Del Deporte, Murcia, v. 2, n. 1, p. 89-99, abr. 2013.

GIL, Alexander, DEL VILLAR, Fernando. Aplicación de un programa de entrenamiento decisional, en tiempo real de juego, para la mejora de rendimiento táctico individual del deportista. En Luis GarcíaGonzález y Fernando del Villar (Eds.), Entrenamiento táctico y decisional en el deporte. Madrid: Síntesis, 2014. p. 132-146.

GONZÁLEZ-VÍLLORA, Sixto et al. Conocimiento táctico y toma de decisiones en jóvenes jugadores de fútbol (10 años). Revista de Psicología Del Deporte, Palma de Mallorca, v. 20, n. 1, p. 79-97, 2011. 
GRAY, Shirley; SPROULE, John. Developing pupils' performance in team invasion games. Physical Education and Sport Pedagogy, London, v. 16, n. 1, p. 15-32, jan. 2011.

GRIFFIN, Linda L.; BROOKER, Ross; PATTON, Kevin. Working towards legitimacy: two decades of teaching games for understanding. Physical Education and Sport Pedagogy, London, v. 10, n. 3, p. 213-223, nov. 2005.

GUTIÉRREZ, David et al. Assessment of Secondary School Students' Game Performance Related to Tactical Contexts. Journal of Human Kinetics, Katowice, v. 42, p. 223-234, 2014.

GUTIÉRREZ, David; GARCÍA-LÓPEZ, Luis M. Assessment of primary school students' decisionmaking related to tactical contexts. Journal of New Approaches in Educational Research, Alicante, v. 1, n. 1, p. 7-12, jul. 2012.

GUTIÉRREZ, David et al. Diferences in decisión-making development between expert and novice invasión game players. Perceptual and Motor Skills, Missoula, v. 112, n. 3, p. 871-888, 2011.

HARVEY, Stephen. A study of U19 college soccer player's improvement in game performance using the Game Performance Assessment Instrument. In: LIGHT, K. Swabey; BROOKER, R. (Ed.). Proceedings of the 2nd International Conference: Teaching Sport and Physical Education for Understanding. Melbourne: University of Melbourne, 2003. p. 11-25.

HARVEY, Stephen; CUSHION, Christopher J.; MASSA-GONZÁLEZ, Ada N. Learning a new method: Teaching Games for Understanding in the coaches' eyes. Physical Education and Sport Pedagogy, London, v. 15, n. 4, p. 361-382, oct. 2010.

HARVEY, Stephen; GITTINS, Christopher. Effects of integrating video-based feedback into a Teaching Games for Understanding soccer unit. Ágora para la Educación Física y el Deporte, Valladolid, v. 16, n. 3, p. 271-290, sept. 2014.

HASTIE, Peter A.; SINELNIKOV, Oleg A.; GUARINO, Anthony J. The development of skill and tactical competencies during a season of badminton. European Journal of Sport Science, London, v. 9, n. 3, p. 133-140, may 2009.

HOPPER, Timothy. Teaching games for understanding: the importance of student emphasis over content emphasis. The Journal of Physical Education, Recreation and Dance, Reton, v. 73, n. 7, p. 44-48, sept. 2002.

LIGHT, Richard L.; HARVEY, Stephen; MOUCHET, Alain. Improving "at-action" decision-making in team sports through a holistic coaching approach. Sport, Education and Society, Oxon, v. 19, n. 3, p. 258-275, 2014.

MAÑERO, Juan; RODRÍGUEZ, Hugo. Propuesta para abordar el qué y cómo enseñar las habilidades motrices en secundaria: un ejemplo a través del patinaje. Ágora para la Educación Física y el Deporte, Valladolid, v. 13, n. 2, p. 197-228, mayo 2011.

NIELSEN, Tjai M.; MCPHERSON, Sue L. Response selection and execution skills of professionals and novices during singles tennis competition. Perceptual and Motor Skills, Missoula, v. 93, p. 541-555, 2001.

OSLIN, Judith L.; MITCHELL, Sthephen A.; GRIFFIN, Linda. The game performance assessment instrument (GPAl): Development and preliminary validation. Journal of Teaching in Physical Education, Champaign, v. 17, p. 231-243, jan. 1998.

PHILLIPS, Elissa et al. Expert performance in sport and the dynamics of talent development. Sports Medicine, Auckland, v. 40, n. 4, p. 271-283, 2010.

ROBLES, José; GIMÉNEZ, Francisco J.; ABAD, Manuel T. Metodología utilizada en la enseñanza de los contenidos deportivos durante la E.S.O. Revista Internacional de Medicina y Ciencias de la Actividad Física y el Deporte, Madrid, v. 10, n. 41, p. 35-57, 2011. 
SERRA, Jaime; GONZÁLEZ-VÍLLORA, Sixto; GARCÍA-LÓPEZ, Luis M. Comparación del rendimiento de juego de jugadores de fútbol de 8-9 años en dos juegos modificados 3 contra 3 . Cuadernos de Psicología del Deporte, Murcia, v. 11, n. 2, p. 77-91, jun. 2011.

SINGLETON, Ellen. From command to constructivism: Canadian secondary school physical education curriculum and teaching games for understanding. Curriculum Inquiry, Toronto, v. 39, n. 2, p. 321342, mar. 2009.

STOLZ, Steven; PILL, Shane. Teaching games and sport for understanding: exploring and reconsidering its relevance in physical education. European Physical Education Review, Melbourne, v. 20, n. 1, p. 36-71, jan. 2014.

TALLIR, Isabel et al. Assessment of game play in basketball. In: LIGHT, K. Swabey; BROOKER, R. (Ed.), Proceedings of the 2nd International conference: Teaching Sport and Physical Education for Understanding, Belgium: University of Ghent, 2004. p. 108-114.

THORPE, Rod; BUNKER, David; ALMOND, Len. A change in focus for the teaching of games. In: PIERON, M.; GRAHAM, K. C. (Ed.) The 1984 Olympic Scientific Congress Proceedings, 6. Sport Pedagogy. Champaign: Human Kinetics. 1986. p. 163-169.

TURNER, Adrian; MARTINEK, Thomas J. A comparative analysis of two models for teaching games: technique approach and game-centered (tactical focus) approach. International Journal of Physical Education, Schorndorf, v. 29, n. 4, p. 15-31, 1992.

WEIN, Horst. Contraatacar con inteligencia: desarrollar la inteligencia en el juego ofensivo. Easy Sports-Software CB/Sportakademie24, 2012. [libro electronico]. ISBN: 978-84-937969-1-4. 